\title{
ESTIMATING BEAM INTENSITY \\ FROM THE BOOSTER BPM SYSTEM
}

\section{BOOSTER TECHNICAL NOTE}

NO. 206

\author{
D. Ciardullo
}

February 11, 1992

\author{
ALTERNATING GRADIENT SYNCHROTRON DEPARTMENT \\ BROOKHAVEN NATIONAL LABORATORY \\ UPTON, NEW YORK 11973
}




\section{ESTIMATING BEAM INTENSITY FROM THE BOOSTER BPM SYSTEM}

\section{INTRODUCTION:}

The Booster BPM system has several intermediate outputs which contain intensity information about the beam. Although not the instrumentation of choice for such a measurement, the BPM system does provide a convenient check of average beam intensity at 46 locations around the ring. A plot of $V_{\text {sum }}$ at every PUE location is available each time an orbit is taken. The primary purpose of this technical note is to relate the BPM system suMs voltages to average beam intensity. In addition, the relationship between beam charge and the various outputs of the front end electronics will be presented.

The position detector used by the system consists of two electrostatic pickup electrodes (PUEs) in the form of a split cylinder. There are several subtle points which make this type of beam sensor useful for measuring beam intensity:

1. The detector senses the amount of charge in the beam, as opposed to current (charge per unit time). This implies measurement signal levels independent of the speed of the beam bunch.

2. With the exception of the small gap between the electrodes, the split cylinder completely encircles the passing beam; The sum of the charge on both PUEs is therefore proportional to the total charge in the beam bunch.

3. The split cylinder has an extremely linear response, making the sum signal independent of the beam position within the detector.

4. PUE signals represent the instantaneous longitudinal charge density of a passing beam bunch, integrated over the length of the electrodes. Since this length ( 0.21 meter) is very small compared to the shortest expected bunch length $(\approx 22$ meters at a bunch factor of 1/6), the point at which the PUE signal is a maximum approximates the peak value of charge in the bunch.

In addition to the above mentioned detector attributes, the front end processing (FEP) electronics are wideband with a high input impedance. This preserves the temporal bunch profile as seen by the PUEs, making the auxilliary analog outputs from the FEP module ideal for peak intensity measurements. 


\section{DEFINITIONS :}

FEP The Front End Processing electronics; This module is the interface between the PUEs and the acquisition electronics, where gain (or attenuation) is applied to the bunch signals.

$\mathrm{C}_{\mathrm{L}} 1 / 2$ the total capacitance load seen by the beam. This includes one PUE, its feedthru, the cable assembly (with thermal isolator) and the equivalent input capacitance of one channel from the FEP module. The following capacitance values are approximate, and may vary from one BPM station to another by as much as $\pm 10 \%$ :

$$
\begin{aligned}
& C_{L}=2700 \mathrm{pf} \text { for } x 0.1 \text { BPM system gain mode } \\
& C_{L}=300 \mathrm{pf} \text { for } \mathrm{x} 1 \text { and } x 10 \text { BPM system gain mode. }
\end{aligned}
$$

$G$ The gain of the FEP amplifiers (but not necessarily the "gain mode" of the BPM system).

$$
\begin{aligned}
& G=1 \text { for } \times 0.1 \text { and } x 1 \text { BPM system gain modes } \\
& G=10 \text { for } \times 10 \text { BPM system gain mode. }
\end{aligned}
$$

$\mathrm{N}$ The number of rf cycles (bunches) over which the real-time sum and difference bunch signals are integrated (also known as $\mathrm{N}$ BUNCH).

$\mathrm{h}$ The harmonic number of the Booster. currently, $\mathrm{h}=3$ for protons and $\mathrm{h}=3$ (or $\mathrm{h}=12$ ) for heavy ions.

B The beam bunching factor $(B \leq 1)$.

$V_{A}(t)$ These two terms represent the real-time bunch $V_{B}(t) \quad$ signals from electrodes $A$ and $B$, respectively, appearing at the auxilliary outputs of certain "special" FEP modules. Each plate signal is linearly proportional to the amount of charge induced onto its respective electrode due to the passing beam.

$V_{D}(t)$ These two signals are proportional to the real-time $v_{S}(t)$ difference and sum of the bunch signals $V_{A}(t)$ and $V_{B}(t)$. They appear at the auxilliary outputs of all "standard" FEP modules.

$Q_{A}$
$Q_{B}$

The total charge induced on plate $A$ or plate $B$, integrated over some specified period of time.

$Q_{A}(t) \quad$ The instantaneous charge induced on plate $A$ and

$Q_{B}(t)$ plate $B$.

$\mathrm{Q}_{\text {BUNCH }}$ The total charge contained in one beam bunch. 
One should refer to this list of definitions when choosing values for $G, C_{h}, h, B, f_{R F}, C_{I N T}$ and $N$ for the equations presented in the following sections.

Figure 1 shows the relationship between the charge on each PUE and the various analog FEP output voltages. The diagram also shows how the BPM system integrator outputs relate to the average charge in each bunch.

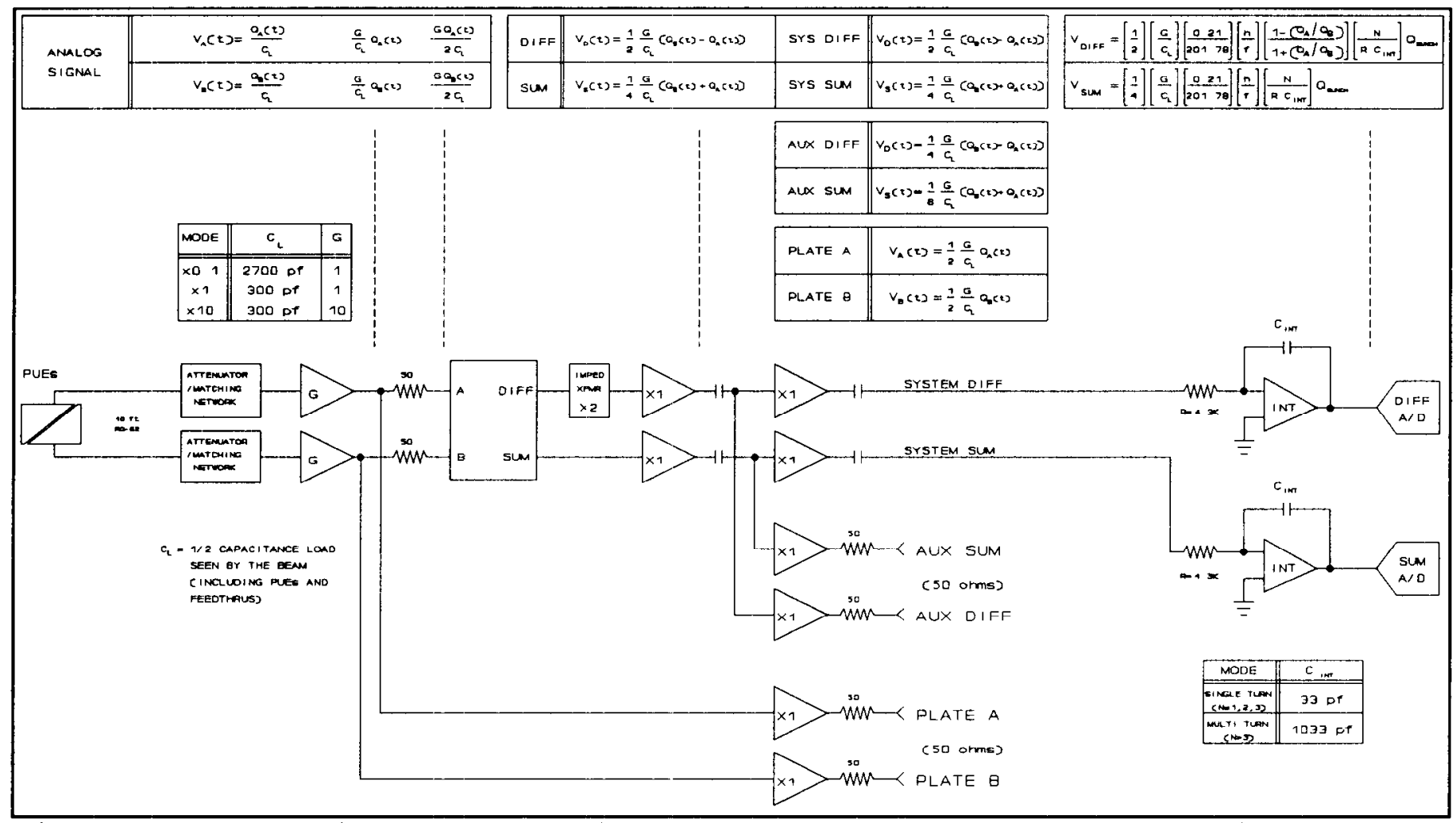

Figure 1. Functional Block Diagram of BPM Front End Electronics.

BPM SYSTEM OUTPUTS (values displayed via "booster_orbit"):

The raw data reported to the booster orbit program (via the BPM Instrument controller) are digitized versions of the integrated sum and difference voltages output by the front end electronics.

$$
\begin{gathered}
V_{D I F F}=\frac{1}{2} \frac{G}{C_{L}} \frac{0.21}{201.78} \frac{h}{f_{R F}} \frac{1-\left(\frac{Q_{A}}{Q_{B}}\right)}{1+\left(\frac{Q_{A}}{Q_{B}}\right)} \frac{N}{R C_{I N T}} Q_{B U N C H} \\
V_{S U M}=\frac{1}{4} \frac{G}{C_{L}} \frac{0.21}{201.78} \frac{h}{f_{R F}} \frac{N}{R C_{I N T}} Q_{B U N C H}
\end{gathered}
$$


where:

$$
\begin{aligned}
& C_{I N T}: 1033 E-12(N \geq 4 \quad \text { - Normal mode }) \\
& 33 E-12 \text { ( } \leq 3 \text { - Single Turn mode) } \\
& \text { G: } \quad 1 \text { ( } x 0.1 \text { and } x 1 \text { gain modes) } \\
& 10 \text { ( } x 10 \text { gain mode) } \\
& C_{L}: 2700 E-12 \text { ( } x 0.1 \text { gain mode) } \\
& \mathrm{h}: \quad \begin{array}{r}
300 \mathrm{E}-12 \text { ( } \mathrm{x} 1 \text { and } \\
\text { harmonic number }
\end{array} \\
& \mathrm{N} \text { : number of bunches integrated over } \\
& \text { R: } 4300 \text { ohms (integrator resistance) }
\end{aligned}
$$

The constants 0.21 and 201.78 represent the length of the PUEs and the circumference of the Booster, respectively, and $\left(f_{R F}\right)^{-1}$ is the integration time for one bunch. The relation for $V_{\text {sum }}$ in Eq. (1) can be solved in terms of $Q_{\text {BUNCH }}$ and used to obtain an expression for the average beam intensity:

$$
\begin{aligned}
\begin{aligned}
Q_{B U N C H} & =4 \frac{201.78}{0.21} \frac{C_{L}}{G} \frac{f_{R F}}{h} \frac{R C_{I N T}}{N} V_{S U M} \\
& =16.5 E 6 \frac{C_{L}}{G} \frac{f_{R F}}{h} \frac{C_{I N T}}{N} V_{S U M}
\end{aligned} \\
\text { INTENSITY }=\frac{h Q_{B U N C H}}{1.6 E-19} \\
=103.3 E 24 \frac{C_{L}}{G} \frac{C_{I N T}}{N} f_{R F} V_{S U M}
\end{aligned}
$$

Eq. (3) can be made more accurate (especially for beam intensities less than $\left.10^{11} \mathrm{ppp}\right)$ by replacing $v_{\text {sum }}$ with $\left(\mathrm{V}_{\text {sum }}-\mathrm{V}_{\text {offset }}\right)$. $\mathrm{V}_{\text {offset }}$ is the value returned by the electronics when an orbit measurement is made with no beam in the Booster. Offsets in both the PUEs and the electronics rob the system of dynamic range, and should be taken into account whenever possible.

If the speed of the beam is known and the $r f$ frequency sweep is "small" during the time in which integration of the bunch signals takes place, then eq. (2) can be used to approximate the average beam current in the Booster:

$$
\begin{aligned}
I_{B E A M} & =\frac{\beta C}{201.78} h Q_{B U N C H} \\
& =24.5 E 12 \quad \beta \frac{C_{L}}{G} \frac{C_{I N T}}{N} f_{R F} V_{S U M}
\end{aligned}
$$




\section{REAL-TIME FEP MODULE OUTPUTS:}

The following paragraphs involve only the FEP module outputs; Any additional gain (or loss) incurred due to the use of fiberoptic links (or long coaxial cable runs) must also be taken into account.

\section{Auxilliary SUM and DIFF outputs}

The standard FEP module used by the Booster Average orbit system has an auxilliary set of SUM and DIFF outputs. The signals obtained at these outputs are proportional to the sum and difference of the longitudinal charge density profile of the beam, integrated over the length of the PUEs.

$$
\begin{aligned}
& V_{D}(t)=\frac{1}{4} \frac{G}{C_{L}}\left(Q_{B}(t)-Q_{A}(t)\right)=\frac{1}{4} \frac{G}{C_{L}} \frac{0.21}{201.78} \frac{h}{B} \frac{1-\left(\frac{Q_{A}}{Q_{B}}\right)}{1+\left(\frac{Q_{A}}{Q_{B}}\right)} Q_{B U N C H}(t) \\
& V_{S}(t)=\frac{1}{8} \frac{G}{C_{L}}\left(Q_{B}(t)+Q_{A}(t)\right)=\frac{1}{8} \frac{G}{C_{L}} \frac{0.21}{201.78} \frac{h}{B} Q_{B U N C H}(t)
\end{aligned}
$$

If the relation for $V_{S}(t)$ in $E q .(5)$ is solved for $Q_{B U N C H}(t)$, the resulting expression shows the peak (instantaneous) beam current with respect to $V_{S}(t)$. Making the substitution $B=\left(t_{0} f_{R F}\right) / 2$, and assuming a $\cos ^{2}$ bunch shape (see Figure 2):

$$
\begin{aligned}
Q_{B U N C H}(t) & =8 \frac{C_{L}}{G} \frac{201.78}{0.21 h} \frac{t_{0} f_{R F}}{2} V_{S}(t) \\
I_{b}(t) & =\frac{\beta C}{201.78} h Q_{B U N C H}(t) \\
& =5.71 E 9 \beta \frac{C_{L}}{G} t_{0} f_{R F} V_{S}(t)
\end{aligned}
$$

Since the bunching factor is not always explicitly known, it has been represented in terms of the full bunch width $\left(t_{0}\right)$ and bunch repetition frequency $\left(f_{R F}\right)$, which is readily obtained from the oscilloscope display. 


\section{Auxilliary Plate A and Plate B Outputs:}

There are several "special" FEP modules with an auxilliary set of Plate $A$ and Plate $B$ outputs. These outputs are in addition to the system Sum and Difference outputs, and provide actual real-time bunch signals linearly proportional to the charge induced on each PUE. Properly scaled, these signals can provide information about the peak bunch current.

If $Q_{A}$ and $Q_{B}$ are the charge induced on electrode $A$ and electrode $B$ respectively, then the voltages appearing at the outputs are:

$$
\begin{aligned}
& V_{A}(t)=\frac{1}{2} \frac{G}{C_{L}} Q_{A}(t) \\
& V_{B}(t)=\frac{1}{2} \frac{G}{C_{L}} Q_{B}(t)
\end{aligned}
$$

The sum of the two PUE signals is constant, and can be used to approximate the peak charge in the bunch. The substitution $B=\left(t_{0} f_{R F}\right) / 2$ is again used:

$$
\begin{aligned}
Q_{B U N C H}(t) & =2 \frac{C_{L}}{G} \frac{201.78}{0.21 h} \frac{t_{0} f_{R F}}{2}\left[V_{A}(t)+V_{B}(t)\right] \\
I_{b}(t) & =\frac{\beta C}{201.78} h Q_{B U N C H}(t) \\
& =1.43 E 9 \beta \frac{C_{L}}{G} t_{0} f_{R F}\left[V_{A}(t)+V_{B}(t)\right]
\end{aligned}
$$

Figure 2 shows how a typical bunch signal from the FEP module auxilliary sum output relates to the amount of charge in the Booster. The bunch shape factor is assumed $\cos ^{2}$, with a uniform charge distribution (both longitudinal and transverse). 


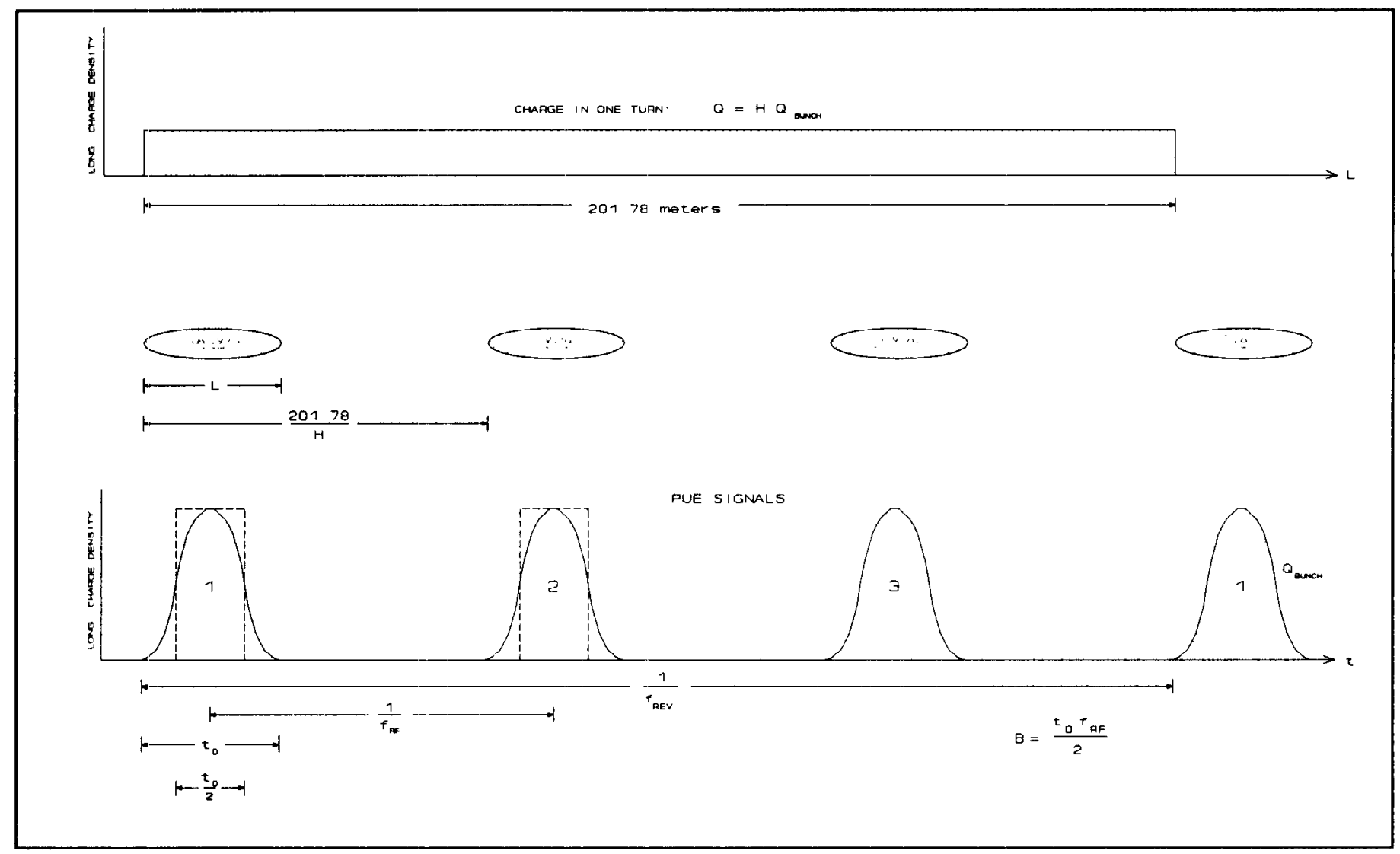

Figure 2. PUE Bunch Signal Example ( $h=3, B=1 / 5)$. 